Revue critique de fixxion française contemporaine

22 | 2021

Figures du mensonge et de la mauvaise foi dans le roman contemporain

\title{
Roman feint et romance vraie dans l'ultracontemporain : Un coup d'un soir de Mathieu Bermann
}

\section{Rodolphe Perez}

\section{(2) OpenEdition}

\section{Journals}

Édition électronique

URL : https://journals.openedition.org/fixxion/367

DOI : $10.4000 /$ fixxion.367

ISSN : 2295-9106

Éditeur

Ghent University

Référence électronique

Rodolphe Perez, «Roman feint et romance vraie dans l'ultracontemporain : Un coup d'un soir de Mathieu Bermann », Revue critique de fixxion française contemporaine [En ligne], 22 | 2021, mis en ligne le 15 juin 2021, consulté le 17 février 2022. URL : http://journals.openedition.org/fixxion/367 ; DOI : https://doi.org/10.4000/fixxion.367

Ce document a été généré automatiquement le 17 février 2022.

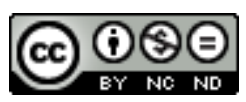

Les contenus de la Revue critique de fixxion française contemporaine sont mis à disposition selon les termes de la licence Creative Commons Attribution - Pas d'Utilisation Commerciale - Pas de Modification 4.0 International. 


\title{
Roman feint et romance vraie dans l'ultracontemporain : Un coup d'un soir de Mathieu Bermann
}

\author{
Rodolphe Perez
}

\section{Introduction}

Dans son dernier roman, Un coup d'un soir, suivi de Dans le lit de Marin, paru en 2019 chez P.O.L., Mathieu Bermann met en scène un écrivain trentenaire qui, par les réseaux sociaux, s'éprend d'un jeune étudiant rennais de vingt ans. Détournant l'autofiction tout en jouant avec ses codes, Bermann inaugure le récit d'un récit qui se construit: le personnage principal rédige un roman sur l'histoire d'amour qu'il vit, dans l'espoir de reconquérir l'amant par lequel il est délaissé. Il cherche alors à sublimer le vécu (au sein de la fiction) afin de susciter le désir du jeune amant. Dans le même temps, il s'agit de produire une œuvre qui ne soit pas vraie, afin de protéger le partenaire officiel du narrateur, Sébastien, de cet adultère secret. De son propre aveu, "c'eût été un mensonge littéraire"1. Il avoue effectivement, à propos de cette supercherie: "En écrivant, je prenais des risques, qui n'en seraient vraiment qu'au moment de la publication" (UC 174). Apparaît une tension entre le dit et l'écrit, renouvelée par une urgence à témoigner de ce qui a eu lieu tout en tentant de protéger l'homme civil des aveux du personnage de fiction. Il ajoute, plus loin: "Le mot roman me protégeait du mieux qu'il pouvait" (UC 174). Ainsi, les deux amants élaborent à leur manière un mensonge de la romance à même d'entretenir un désir dont ils ne perçoivent pas la fin. Le second texte, Dans le lit de Marin vise à retracer l'écriture du texte-cadre dans une sorte d'anthropologie de la relation et afin de faire mentir le titre du premier texte: "coup d'un soir". Le narrateur manœuvre par bien des manières afin de renouveler la nuit des amants et de poursuivre la conquête du désir quand le jeune homme, sans mettre un terme à l'histoire, semble freiner la réalisation du désir. La fiction et la mauvaise foi servent ainsi de prétexte à moduler une réalité décevante : 
On ne se connaissait pas, Marin et moi, on vivait à des centaines de kilomètres ; mais on s'était retrouvés à Rennes pour y passer la nuit ensemble. Nous n'en avions pas eu d'autres, du moins à l'heure où j'avais terminé d'écrire - mais la suite, qui m'était alors inconnue, ne présageait rien qui aille dans un sens contraire. (LM 191)

L'enjeu est ainsi celui d'une seconde nuit, afin d'affirmer le mensonge de la première, quitte à truquer le réel et à révéler la supercherie d'une disparition du désir. Ainsi, le récit apparaît comme une manipulation féconde qui nourrit la vraisemblance, tout en subsumant le réel. Aussi, la fiction seconde visant à instituer un consensus du désir là où les deux protagonistes ne s'accordent pas, l'œuvre double de Bermann rejoint-elle la définition du mensonge élaborée par Jochen Mecke: “Tout mensonge consiste en une divergence entre un sentiment et une opinion d'une part et une énonciation ou une expression d'autre part"'. Le roman qui raconte la scène amoureuse, tout en cherchant à dépasser cette divergence, s'impose comme l'expression première et l'affirmation du sentiment du narrateur contre l'opinion du jeune amant. Celle-ci s'inscrit comme preuve factice du vrai.

\section{Faire mentir la fin}

3 Le risque contre lequel lutte le personnage du narrateur est celui d'une désillusion du désir, laquelle pourtant est une réalité. Dans son opérabilité, le désir ne peut que se solder par un échec puisque les deux personnages n'aspirent pas à la même relation. Ce postulat ouvre la voie à la manifestation d'une mauvaise foi évidente de la part du narrateur puisque, s'y refusant, elle nie cette réalité factuelle ${ }^{3}$. Il s'agit pour lui de construire une croyance ferme en ce désir, c'est-à-dire de fictionnaliser sa réalité.

"De bonne ou mauvaise foi, je persévérai à rechercher le désir de Marin. Comme un objet perdu qu'on s'imagine pouvoir recouvrer" (LM 194). Ainsi, le pouvoir d'imagination de l'écrivain, en ce qu'il appuie une fiction de la romance, permet de pallier l'insuffisance du réel ; la fiction s'érige sur cette mauvaise foi confirmée :

Il n'empêche que, secrètement, je ne parvenais toujours pas à croire à la perte définitive de son désir. Et s'il continuait d'afficher son refus, celui-ci me paraissait douteux. Marin avait dit NON. Mais dans le fond, cela voulait peut-être dire autre chose. Les mots pouvaient être trompeurs - j'étais bien placé pour le savoir car, au contraire de ce que j'avais dit, je ne voulais pas voir Marin juste pour dormir. Il s'ensuivit que je ne consentis pas le moins du monde à son absence de consentement. (LM 194-195)

5 L'enjeu central au cœur du second récit est bien celui d'une seconde nuit des amants, fantasme absolu dont le personnage cherche la répétition. Or, ce refus de "croire" à la réalité d'un refus de Marin l'invite à amorcer des manœuvres pour faire flancher le jeune homme, c'est-à-dire à scénariser la romance pour diriger le jeu de l'amant. L'absence de consentement qu'évoque Bermann manifeste très clairement le refus du réel de son personnage de romancier et l'élaboration progressive d'une mauvaise foi active puisqu'elle préside à son comportement démiurgique ${ }^{4}$. Dans le même temps, le texte tire vers l'autofiction, là où l'on pourrait considérer le second livre comme récit vécu du personnage du premier roman. L'écriture s'élabore alors autour de cette "divergence" et de son expression, ou de deux manières de voir le réel. Alors que Marin prétend en amont vouloir dormir avec le narrateur mais sans coucher avec lui, ce dernier caresse le sexe de son partenaire. Ainsi, Marin s'insurgera plus tard d'un geste qu'il avait prévenu. Et le narrateur de confier : "Certes Marin ne mentait pas sur ce qui 
s'était passé; mais je n'aurais pas menti, moi non plus, en racontant les choses différemment" (UC 196). C'est bien l'écart entre deux sentiments du réel qui est l'enjeu principal, qui se place à la frontière des mensonges et est moteur de la mauvaise foi. Le narrateur semble prendre conscience d'une altérité qui fissure la finitude supposée de l'histoire. Ce geste est pourtant précisément permis par sa mauvaise foi. Effectivement, il confie en amont à propos de Marin :

Il m'annonça que nous ne coucherions plus ensemble. Pourtant prévisible, cette déclaration me laissait effaré.

Le livre en était le contrecoup. Je l'avais écrit dans l'urgence. Au fond, je l'avais écrit dans une certaine méconnaissance de ce qu'était réellement cette histoire. (LM 193)

6 Aussi, malgré le pressentiment juste d'une fin de l'histoire, le narrateur agit en refusant cet état de fait. Il ajoute d'ailleurs: "Justement, j'avais écrit Un coup d'un soir pour en faire mentir le titre, c'est-à-dire l'ordre des choses tel qu'il se dessinait désormais" (LM 193). L'œuvre de fiction entend combler ce que le réel ne permet plus, ce que le réel a d'inacceptable pour le narrateur, il est le produit pur de sa mauvaise foi à l'égard du réel.

7 Se résoudre à écrire un roman signifie, pour le narrateur, que la fiction constitue une échappée. Le refus d'accepter la mort de la relation implique de lui donner une réalité interminée dans la fiction, mais pas pour en marquer le deuil, ce que l'écriture permet souvent. Ce procédé, non plus du deuil mais de la permanence d'une réalité de l'absence - donc de son mensonge - préside par exemple à l'autofiction Fou de Vincent d'Hervé Guibert, qui s'ouvre sur la mort de Vincent dont pourtant l'auteur dévoile la présence. L'écriture lui permet donc de croire à la continuité de l'histoire alors même que la rédaction du roman a pu se faire parce que l'histoire était finie : l'écriture est malgré elle la vérité de l'absence. Le livre est la preuve matérielle d'une fictionnalité de la permanence d'autrui alors même que "tout mensonge qui se révèle lui-même comme tel n'en est pas un" vérité du moi, vérité référentielle, plus qu'une vérité de fiction. La première visée est celle d'une cristallisation de l'évanescence du désir, elle est rapidement dépassée par l'appétit d'une perpétuation de ce désir dans le retour de la scène originelle. Ainsi, désormais, l'imagination du narrateur va sans cesse chercher à contrecarrer la réalité du refus de Marin, puisqu'il l'affirme simplement: “La disparition du désir, je n'y croyais pas" (UC 193). Quelque temps après, lors d'un échange de SMS, alors que Marin se montre plutôt froid et distant depuis la fameuse nuit, le narrateur lui demande s'ils peuvent se téléphoner. Marin répond "Je peux répondre", oubliant la négation: "Je peux pas répondre, corrigea Marin, par message à la suite de mon appel" (LM 210), un appel auquel il n'a pas répondu. Et le narrateur de déployer une rhétorique du lapsus : "Je plaidai ma propre cause, celle de mon désir encore intact, en prétendant que c'était son inconscient qui parlait" (LM 211). Ainsi, tout devient prétexte à assigner le réel à la fiction du désir définie par le narrateur. Pourtant une intuition persiste, même si elle ne l'empêche pas de continuer : "A travers la débâcle de la conversation, je lisais sans le vouloir la faillite certaine de son désir pour moi” (LM 211). Il y a bien une décrue du désir de Marin, imposée par les faits, qui engage le narrateur à poursuivre son refus d'y croire afin de consolider sa manœuvre. En effet, il n'abandonne. L'été s'achève. "Entretemps, Marin avait déménagé de Rennes à Paris, où il était désormais en alternance, tour à tour étudiant et salarié. Je me proposais de l'y retrouver, sous un faux prétexte" (LM 212). Se dessine alors progressivement la bipolarité de la mauvaise foi du narrateur : d'une part, se trouve une mauvaise foi réflexive, dans son refus d'accepter 
la fin de la séduction, d'autre part, se trouve une mauvaise foi à l'égard du jeune homme, un mensonge qui appuie son argumentation, ici sous le jour du "faux prétexte": "Je laissais entendre que je pouvais changer la date de ma venue, dont le motif affiché n'était qu'un faux-semblant" (LM 213). En d'autres termes, le réel montré, "affiché", sert d'argument pour un réel espéré, et le mensonge tient alors lieu de fiction qui ménage la scène du réel. Il en est de même du livre qui, parallèlement aux tentatives de rencontre physique, joue à entretenir la fiction du désir : "Le livre était désormais fini, sans que j'en aie fini avec l'illusion qu'il puisse changer le cours des choses lorsque Marin le lirait" (LM 213-214). L'hypothèse que Marin lise le texte prend l'allure d'une certitude - d'un projet - afin d'encourager la légitimité de l'érotisme, tout en restant la preuve de sa virtualité :

Mais le livre était un mirage qui persistait dans mon esprit: toutes ces pages que j'avais écrites sur Marin me semblaient un véritable attrait, susceptible de ressusciter celui que Marin avait eu pour moi, et qu'il n'avait plus.

Aussi m'adonnai-je à des confidences calculées. (LM 214)

8 Livre-prétexte, livre-manœuvre, il tend à médiatiser le désir. "Mirage", il a vocation à troubler la perception du jeune homme, à le faire tomber dans le camp de l'irréel pour que s'éprouve la réalité du désir, celui du narrateur. Dans son ouvrage intitulé Façons de lire, manière d'être, Marielle Macé élabore une réflexion sur la place de la lecture dans la littérature et dans le rapport au texte. Puisque l'un des enjeux de l'œuvre de Bermann réside bien dans l'élaboration d'un roman comme preuve du désir afin de susciter une lecture imitative du personnage de l'amant, on peut s'interroger sur les spécificités du rôle que joue le livre dans le rapport à la réalité, ici entendue comme fiction de la réalité, doublée de la fiction du livre. Marielle Macé ouvre en ces termes: “Il n'y a pas d'un côté la littérature et de l'autre la vie, dans un face-à-face brutal et sans échanges

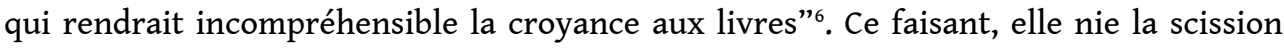
opérée entre le réel et la fiction, de la même manière que semble l'envisager le personnage du narrateur. Ce dernier compose un livre qui vise à jouer le rôle de passerelle entre la réalité vécue par le personnage et la fiction, fiction romanesque et fiction du fantasme. Le livre, tel qu'il s'élabore, prend la valeur d'un échange symbolique, d'une invitation faite par le narrateur, invitation réflexive mais invitation également à l'égard de l'amant, à croire, ou, pour reprendre Coleridge cité par Jochen Mecke à une "suspension of disbelief", pour s'ouvrir à la possibilité offerte par la fiction. Ainsi, le livre endosse un rôle de trouble du réel, il se présente comme l'autorisation d'une négation d'une réalité opaque. Il s'agit donc de refuser "un face-àface qui ferait [...] une simple confusion entre la réalité et la fiction, un renoncement à l'action, une humiliation du réel, et par conséquent un affaiblissement de la capacité à vivre" ( $F L$ 10). On sent bien combien, dans le roman de Bermann, les fluctuations du réel à la fiction imposent une telle lecture: la fiction vient au secours du réel, une fiction qui se nourrit du réel quand le réel est brouillé par la fiction. Il y donc une porosité entre les deux, que matérialise d'ailleurs l'enchevêtrement des romans, du récit cadre au récit de fiction. De la même manière, cette porosité est doublée par la virtualisation des échanges entre les personnages : c'est par le réseau Instagram que les échanges débutent, par une fiction de soi qui séduit l'autre et initie au désir, à la rencontre et à la conquête de ce même désir fugace. On pourrait dire, reprenant la remarque de Macé, qu'ici, le réel ne sort pas humilié de la démarche mais qu'il tend à être vivifié par les possibilités qu'offre la fiction. Et d'ajouter: "Il y a plutôt, à l'intérieur de la vie elle-même, des formes, des élans, des images et des manières d'être 
qui circulent entre les sujets et les œuvres, qui les exposent, les animent, les affectent" (FL 10). Le personnage du narrateur triche avec le réel dans une volonté de pallier ses failles grâce au mensonge du roman.

La spécificité du héros chez Bermann réside dans le fait que ce n'est pas son rapport à la lecture livresque qui brouille la réalité référentielle mais son rapport à l'écriture : le réel qu'il vit devient un moteur d'écriture qui s'envisage comme arme pour affecter le réel. Ainsi, l'écriture s'entend comme une lecture de soi, une interprétation, et opère un mouvement continu entre le réel de la fiction, celui que vit le personnage, et la fiction du réel de la fiction, celle qu'il compose dans l'œuvre. Il y a et "pacte fictionnel et pacte référentiel" $\mathrm{s}$ le texte joue toujours sur la possibilité de l'autofiction. Le texte cadre serait l'univers de référence du texte de fiction. Le roman expose l'évolution du personnage, modifié par son réel, reprenant ses droits sur ce réel par le biais d'une création fictionnelle. Le travail réflexif qu'il débute aboutit à un refus d'accepter le réel, celui d'une fin du désir, pour le transmuer, dans un nouveau rapport de soi au réel. De cette manière, cette lecture du moi par lui-même, dans ce qu'elle tente d'entraver du réel, se transmet ensuite à l'amant, devenu personnage de fiction. Alors, si une lecture et une posture de lecteur devaient être définies dans le roman, ce serait du côté de l'amant: l'œuvre écrite dans la fiction a vocation à être lue par Marin et, se faisant, à agir sur sa conduite amoureuse. Ainsi, l'œuvre de fiction vise à détourner son lecteur, assumant totalement sa potentialité subversive, à rebours des critiques des dangers de la lecture dont elle endosse ici le paradigme positif. En somme, le héros fait le pari d'une bovarysation de son lecteur-amant qui, s'éprenant de l'idylle racontée, fût-elle déjà vécue, fût-elle déjà la sienne, chercherait à revivre la nuit des amants, réel référentiel: qu'épris de l'histoire, il s'éprenne à nouveau de l'écrivain; qu'épris du roman, il déplace son désir médiatisé sur son créateur, bref que s'engage un transfert, un déplacement de l'Eros. Il cherche donc à susciter une imitation de la scène fictionnelle, duplicata du "coup d'un soir" dont il regrette la fin. Disons donc que le narrateur fait le pari de diriger son personnage, pour diriger l'homme, dans une invitation à la répétition.

\section{Médiatiser le désir}

10 Aussi, pour reprendre une conception girardienne, le narrateur cherche-t-il à trianguler le désir afin de maintenir dans sa fiction l'élan du jeune Marin. Il lui désigne un modèle, médiateur du désir, qui n'est que le personnage de lui-même, modèle à suivre donc, et se distingue lui-même comme projection de l'accomplissement du désir. Or René Girard précise la menace qui pèse sur un tel projet d'identification : il ouvre la voie à une possible déperdition dans le désir de l'autre. Il existerait ainsi une possibilité pour le narrateur d'écouter le désir de Marin, nouvelle manifestation d'une mauvaise foi, puisqu'elle viendrait précipiter la faillite du retour espéré du désir initial ${ }^{9}$ :

La médiation engendre un second désir parfaitement identique à celui du médiateur. C'est dire que l'on a toujours affaire à deux désirs concurrents. Le médiateur ne peut plus jouer son rôle de modèle sans jouer également, ou paraître jouer, le rôle d'un obstacle. ${ }^{10}$

11 L'enjeu est conséquent. Marin, s'il venait à désirer être le Marin de fiction, et à s'offrir à l'éternité de l'amour, n'aurait qu'une issue : vivre pleinement l'amour avec l'être qu'il désire, c'est-à-dire l'écrivain. Mais l'écrivain ne peut tolérer que son réel soit affecté 
outre mesure: il ne mettra jamais fin à son couple pour vivre avec Marin, issue pourtant légitime, si l'on peut dire, et souhaitée par un Marin qui serait amoureux. Le triangle mène ainsi à une impasse, impasse surtout pour le narrateur qui fait mine de ne pas la voir, refusant le réel référentiel lorsqu'il le gêne, lui préférant une fictionnalisation. Ainsi, ce statut factuel de la romance s'érige en mensonge, et donc en "moyen de se soustraire aux normes et aux valeurs dominantes et à leur contrôle permanent sans risquer toutefois une sanction immédiate" ${ }^{11}$. La réalité serait cette sanction, face aux valeurs de fidélité et de sincérité dans le couple. Le narrateur ménage l'intime et le public : introduire le mensonge sert à éviter la responsabilité. La mauvaise foi dans laquelle il s'engouffre l'autorise à croire qu'il pourrait vivre cette relation secrète, entretenir sa fiction, sans que cette fiction ne vienne saboter sa relation conjugale. Le narrateur cherche donc à être désiré par l'amant dans les seules limites de son propre désir secret. Dès lors, inviter Marin à médiatiser son désir à travers son double fictionnel ne peut que se solder par un retour de la nuit des amants dans les mêmes modalités : fugace, éphémère et donc insatisfaisante. L'éternel retour de l'éphémère, pari que fait le narrateur, le maintient en tension sans jamais abattre l'obstacle. Finalement, la fiction permet au narrateur d'espérer cette bovarysation de Marin et maintient l'excitation d'un interdit dans une volonté de super-imitation, reconduction du même absolu, dans l'espoir inavoué ou inconscient d'une même finalité : l'impossible de la relation.

12 Marin semble avoir pressenti une telle démarche puisqu'il tente de couper court à plusieurs reprises au retour de la nuit des amants. Il se maintient en objet de désir mais se pare du bénéfice de l'interdit. Il affirme que les amants peuvent se voir sans consommer l'histoire, prétextant une relation déplacée sur le régime de l'amitié, ce que rien ne laisse évidemment présager, comme s'il jouissait de se savoir désiré. S'opère ainsi un renversement fécond : si le narrateur cherche à établir une dialectisation selon laquelle le Marin réel viendra à dépasser le Marin fictionnel pour dépasser sa volonté de faire couple en ne s'abandonnant qu'à son rôle d'amant, c'est lui-même qui se retrouve piégé par la fiction puisqu'il bute contre une perpétuelle frustration. Sauf à faire l'hypothèse d'une jouissance pure de l'écriture et de la frustration, ou de la frustration comme moteur de la création. Dans ce cas, la mauvaise foi serait bien plus large et égotiste, et l'idée d'une jouissance de l'écriture ferait de Marin un personnage de fantasme absolu, dénué de toute individuation mais remplissant un rôle. Le narrateur devient prisonnier de sa projection d'un moi écrivain et assujettit l'ensemble de ses manœuvres à un fantasme du moi.

Quoi qu'il en soit, c'est bien le personnage du narrateur qui se retrouve enfermé dans son propre fantasme d'un moi-désirant: désirant l'amant, désirant l'écriture et désirant le désir. À l'inverse, Marin semble échapper à l'implacable dialectisation à l'œuvre : il désire, connaît son désir et ne veut que l'accomplissement de ce désir dans les modalités qu'il a convenues. Le souhait que Marin se projette - relation miroir et imitation - dans son double de fiction, finit par enfermer le narrateur dans la quête de son propre reflet, prisonnier de sa mauvaise foi, c'est-à-dire ici du manque d'authenticité de la scène fictionnelle, ou du manque de potentialités offertes par un réel insatisfaisant. Un point toutefois tend à les rassembler: la manifestation d'une recherche de totalité de l'autre. Le narrateur veut Marin, dans le rôle défini de l'amant. Marin veut le narrateur, d'où sa jalousie à l'égard du conjoint, d'où aussi l'apparition de l'autre amant de Marin, prétexte pour ne pas recoucher avec le narrateur. Ainsi, la 
triangulation romanesque se double d'une triangulation amoureuse. C'est ce qu'on retrouve chez Girard quand il reprend les sentiments de jalousie et d'envie dans son analyse :

La jalousie et l'envie supposent une triple présence : présence de l'objet, présence du sujet, présence de celui que l'on jalouse ou de celui que l'on envie. Ces deux "défauts" sont donc triangulaires : jamais, toutefois, nous ne percevons un modèle dans celui que l'on jalouse parce que nous prenons toujours sur la jalousie le point de vue du jaloux lui-même. Comme toutes les victimes de la médiation interne, celui-ci se persuade aisément que son désir est spontané, c'est-à-dire qu'il s'enracine dans l'objet et dans cet objet seulement. (MR 26)

Dans le cas de Marin, celui qui est jalousé est le conjoint officiel, non pas en tant que tel mais en raison de la position qu'il occupe. Ce dernier est régulièrement invoqué comme une raison pour se refuser au narrateur. Finalement, cette jalousie permet de conserver une triangulation et freine l'entrée de Marin dans le rôle que lui assigne le narrateur. Dans le cas du narrateur lui-même, il n'est pas question de jalousie mais bien d'envie: l'envie est évidemment dirigée vers le jeune homme. Lorsque ce dernier explique être en couple et ne pouvant, de fait, pas dormir avec le narrateur, cela ne suscite pas de jalousie mais demeure un argument dans la perpétuation de la triangulation du côté de Marin. L'envie du narrateur réside dans l'appétit de son désir et dans sa volonté de voir se superposer Marin et le personnage de l'amant. L'objet de la jalousie apparaît comme "un intrus, un fâcheux, un terzo incommodo qui vient interrompre un délicieux tête-àtête" (MR 26). Mais c'est aussi Marin lui-même qui, objet du désir, fait également office de "fâcheux" pour le narrateur, ce qui implique la nécessité de moduler son individualité - son réel - par le biais de la fiction. Le narrateur se dirige en tout cas vers cette démarche : "Pour ma part, j'étais avec Sébastien et cela ne me dérangeait pas le moins du monde qu'on soit ainsi à égalité - comme cela ne m'avait pas vraiment gêné, à Rennes, qu'il soit célibataire et moi pas" (UC 171). La perception de Marin demeure celle qui freine la scène érotique. Si, ici, le réel référentiel du couple ne "gêne" pas le narrateur, ce n'est que pure mauvaise foi puisque l'occasion d'un devenir-référentiel du livre (par sa parution) inquiète et trouble, rappelant que la littérature est "une forme de communication non-mensongère" ${ }^{12}$.

\section{Rhétorique du mensonge}

Cette fragile négation de sa singularité constitue un enjeu de la mauvaise foi du narrateur qui privilégie une authenticité de la fiction à une authenticité de la réalité. Cette perspective manifeste la déréalisation à l'œuvre puisque la relation des amants se cantonne à son souvenir et à sa perpétuation fictionnelle. La mauvaise foi se joue désormais à l'égard du réel. Dans une sorte d'accord implicite entre les différentes parties, qui fonde la triangulation du désir, Girard recourt à la métaphore procréatrice. Une telle démarche a le mérite d'éviter l'écueil du jugement moral où tel personnage serait la dupe de l'autre. Il y a une collaboration des personnages dans le maintien de cette tension.

"L'imagination du héros est la mère de l'illusion mais il faut encore un père à cet enfant et ce père est le médiateur" (MR 36). Ainsi, le narrateur manœuvre la romance, puisqu'il préside à l'illusion, mais cette illusion ne peut tenir que parce que Marin consent à la médiatiser. Les deux amants entretiennent donc la facticité et l'impossibilité de l'Eros, assurant la permanence de sa tension, voire de son interdit. Et c'est parce que Marin 
résiste que le narrateur lui-même perpétue sa mise en jeu dans un esprit de conquête, à la faveur d'un désir égotiste : “je ne voulais pas qu'il me croie rabaissé - ni par l'énoncé de mon désir, ni par l'absence du sien" (LM 218). Aussi, face à la froideur de Marin, il confie : "Sans aucun doute puérile, ma réponse dévoilait ce que j'avais pourtant masqué à ses yeux aussi bien qu'aux miens [...] : une blessure d'amour-propre" (LM 218). Le personnage du narrateur admet ici le principal écueil contre lequel il butte: un aveuglement féroce, un appétit à ne pas abandonner. La mauvaise foi et la rhétorique du mensonge viennent ainsi soutenir un amour-propre mis à mal par le refus du jeune homme. Le narrateur est ainsi balloté entre une réelle attraction érotique et une preuve égotiste de sa capacité à être: "Mais dans cette histoire, l'amour-propre ne m'était pas une ambition durable. Je mettais un certain orgueil à ne pas en témoigner. Toute honte bue, j'avais encore soif de coucher avec lui et j'arrivais parfois à en cacher le désespoir par l'humour" (LM 219). L'humour tout comme la rédaction du roman viennent soulager le regard sur soi, mis à mal par le refus. De la sorte il s'agit de taire ce qui témoignerait d'une forme de faillite de soi par l'orgueil en se concentrant sur l'élaboration du désir. Or, cette volonté de "ne pas en témoigner" assoit d'autant plus la mauvaise foi en ce qu'elle nie précisément l'authenticité émotionnelle du narrateur aux yeux du jeune Marin. Cette détermination est clairement affirmée :

La vérité, je n'en avais que faire: je voulais coucher avec lui, et tant pis si telle n'était pas la vérité absolue de son désir. Marin était bien capable de mentir par omission, qu'il mente donc franchement, pour me faire plaisir tout de bon, puisque ses excuses visaient ostensiblement à m'épargner le déplaisir de son refus. (LM 227)

Dans un habile retournement rhétorique, et dans un souci d'auto-persuasion, le narrateur délibère avec lui-même: Marin sait donc mentir, et en ce sens il est un partenaire de jeu idéal. Mais sachant mentir, il s'en sert pour ne pas éconduire son amant, ce qui certes vise à ne pas froisser son amour-propre mais réduit son importance. Autant qu'il mente donc tout à fait en se glissant dans le rôle de l'amant qui désire. Une telle rhétorique, révélatrice de la capacité tortueuse de l'esprit à se chercher des vérités, témoigne de manière exemplaire d'un refus capricieux d'accepter le réel et surtout d'assumer une responsabilité. Elle a le mérite de montrer combien Marin est bien plus qu'un amant hésitant. Puisqu'en effet, ce qui contrarie le plus le narrateur, c'est l'inaction dans laquelle Marin le place :

Plus que tout m'exaspéraient les excuses derrière lesquelles il se retranchait sans cesse. S'il m'en coûtait d'assumer mon désir, je voulais que Marin fasse de même avec le désir qu'il n'avait plus pour moi. Il n'y avait pas de raison que le refus lui soit moins difficile à formuler qu'à moi de l'entendre. (LM 226-227)

18 Il semble évident que le reproche qu'il formule à l'encontre de Marin - chercher des excuses - est précisément son propre mode opératoire : lui-même se cherche des excuses pour entretenir le désir et le consommer. Marin, acculé, finit par lâcher la sentence: "Je suis désolé, mais je ne ressens plus aucun désir pour toi" (LM 227). Pourtant, un tel verdict ne coupera pas court aux échanges, ni même à la soif de conquête. Marin affirme sa subjectivité, c'est-à-dire qu'il explicite une "divergence", pour reprendre la définition du mensonge de Jochen Mecke, appuyée par des principes : lui, se refuse à la relation adultère :

Malheureusement, en effet, Marin était un garçon à principes. Plusieurs fois, qui toutes m'avaient été désagréables, il avait répété depuis que je le connaissais: “j’ai des principes." Parmi lesquels il y en avait un qui m'avait rendu fou : qu'il ne veuille pas (quand nous ne l'avions pas encore fait) ou qu'il ne veuille plus (alors que nous l'avions déjà fait) coucher avec moi parce que j'étais en couple. (LM 291-292) 
Argumentant en ce sens, Marin ne fait que poser un interdit de plus sans éconduire tout à fait son amant. Ainsi, quelques temps plus tard, le narrateur récidive, se rend à Paris, et utilise un "appât" pour séduire le jeune homme :

Parce qu'il était mythique, et que le hasard voulait que j'y aie mes entrées pour quelque temps, le club de David Lynch [le Silencio] me servait d'appât. On en avait déjà discuté et c'était un bon chantage pour obtenir une nuit avec Marin - même si je savais qu'il ne se passerait rien, en tout cas rien de sexuel. Mais pour que ce chantage, qui ne disait pas son nom, le dise encore moins, je laissai à Marin la possibilité d'un refus qui, lui non plus, ne dirait pas son nom. En effet, je l'avais déjà trop entendu déclarer qu'il n'avait plus de désir pour moi. (LM 260-261)

Déclaration entendue donc mais qui ne semble pas suffire à calmer la manœuvre, ici à son paroxysme. D'abord, on sent bien combien le narrateur cherche à prendre une certaine hauteur intellectuelle, à s'assurer une posture - pour gonfler l'amour-propre? - dans la perspective de séduire le jeune homme. Ensuite, il ménage la possibilité d'un refus pour mieux la contrecarrer et s'assurer une forme de réussite. Enfin, il affiche clairement, au lecteur du moins, sa rhétorique du mensonge afin d'asservir le jeune amant au rôle désigné. Et Marin finit par accepter qu'ils dorment ensemble, admettant : “Je suis sûr qu'il ne se passera rien. Enfin, j'en suis sûr à 99\%" (LM 267). Il ménage ainsi la faille parfaite dans la certitude du narrateur, à partir de laquelle il s'autorisera à caresser son sexe. L'histoire ne dit pas si Marin et le narrateur se sont revus ; toutefois, le jeune homme continue quelques temps de solliciter l'écrivain, comme pour à son tour susciter la tension et l'attention.

\section{Conclusion}

21 Ainsi, chacun se raconte des histoires pour conserver sa vision subjective du désir. Il s'agit de sceller un mensonge, d'affirmer une mauvaise foi pratique mais il s'agit également de définir une vérité de la fiction comme ce qui empêche la fin de l'histoire. Le mensonge, c'est-à-dire l'alibi, se double d'une frustration intérieure menaçante : le réel insatisfait. La mauvaise foi consiste alors à se convaincre que ménager une scène de fiction au désir suffira à maintenir ce désir alerte, au risque que chacun se perde dans son personnage. Cette vérité est définie par Roland Barthes dans les Fragments d'un discours amoureux comme "tout épisode de langage rapporté à la 'sensation de vérité' que le sujet amoureux éprouve en pensant à son amant"13. S'il est rarement question d'amour ici, il y a bien un désir amoureux et érotique de l'autre, et chaque personnage affirme sa "sensation de vérité", c'est-à-dire sa subjectivité de désir, laquelle vise à contraindre l'autre pour aboutir à la scène désirée. Cela se fonde sur une prétention à la dialectisation de l'autre: "l'autre est mon bien et mon savoir : moi seul le connaît, le fait exister dans sa vérité" (Fr 271). Aussi, le narrateur considère-t-il Marin comme son "savoir" et manœuvre-t-il à la faveur de cette certitude fondée sur une négation du réel, une certitude de mauvaise foi : en ce sens s'engage sa lutte conquérante. Il s'agit, par le biais de la fiction, de définir une vérité de Marin, fût-elle alternative. La scène du désir opère autour de cet affrontement des subjectivités et de la capacité du sujet désirant à déformer à sa propre fin l'individualité, ou comme le dit Barthes : “Toujours le même renversement: ce que le monde tient pour objectif, je le tiens, moi, pour factice, et ce qu'il tient pour folie, illusion, erreur, je le tiens, moi, pour vérité. C'est au plus profond du leurre que vient se loger bizarrement la sensation de vérité" (Fr 272). Variation sur le "mentir-vrai" d'Aragon, cette définition de Barthes manifeste sur la 
scène érotique la capacité du sujet désirant à faire plier le réel à sa "sensation" de vrai. Elle affirme de la même manière cette cohabitation efficace de la fiction et du réel, évoquée par Marielle Macé. Ainsi, le caractère fictionnel du désir, du moins comme tentent de l'élaborer chacun des deux amants, s'enracine dans le subterfuge non pas comme une fuite du réel mais bien comme un enrichissement de ce dernier. Ce qui apparaît comme mensonge ne l'est que dans la manœuvre mise en scène pour conquérir l'autre. Si le narrateur est le personnage principal et qu'il n'a aucune omniscience à l'égard de son personnage, puisque le roman se veut réaliste, il lui offre une continuité dans la jouissance d'une fiction. Toutefois, Marin lui-même élabore son désir par le biais d'une autre fiction : la virtualité permise par la distance, tant par les messages Instagram qui augurent la romance, par les photographies attrayantes qu'il envoie de lui que par les messages qu'il écrit pour relancer l'attention du narrateur malgré son refus de consommer le désir. Autant de moyen pour affirmer une facticité de la relation, le primat d'un désir du désir sur sa réalisation. Chacun s'enferme ainsi dans une médiatisation de l'autre, niant une part de sa réalité, se cachant dans un mensonge quant à sa subjectivité, afin d'affirmer l'autorité de son propre désir.

\section{NOTES DE FIN}

1. Mathieu Bermann, Un coup d'un soir, suivi de Dans le lit de Marin, Paris, P.O.L., 2019, p. 172 ; dorénavant $U C$ et $L M$.

2. Joachen Mecke, "Esthétique du mensonge", Cahiers d'Études Germaniques [En ligne], n 68, 2015, mis en ligne le 17 décembre 2017, disponible sur <http://journals.openedition.org/ceg/1436> (consulté le 15 avril 2021).

3. Voir la définition donnée par Sartre de la mauvaise foi, expliquant que cette conduite résulte du désir du sujet d'échapper au mode d'être qui est le sien (L'Être et le Néant. Essai d'ontologie phénoménologique, Paris, Gallimard, 1976 [1943], <Tel>, p. 87-91).

4. Sur le rôle moteur de la mauvaise foi dans l'écriture et sur sa capacité à réinventer l'être, on renverra à l'analyse de Maxime Decout, En toute mauvaise foi, Paris, Minuit, 2015, <Paradoxe>.

5. Jochen Mecke, art. cit.

6. Marielle Macé, Façons de lire, manière d'être, Gallimard, 2011, <Essais>, p. 9, ; dorénavant FL.

7. Samuel Taylor Coleridge, cité par Jochen Mecke (art. cit.), Biographia Literaria, II, Oxford, Clarendon Press, 1907, p. 6.

8. Jochen Mecke, art. cit.

9. Dans En toute mauvaise foi, Maxime Decout montre comment la mauvaise foi éclaire les relations de nombreux personnages dans les textes selon une dynamique du désir qui n'est pas sans rappeler celle qu'étudie René Girard (op. cit., p. 31-49, 68-78).

10. René Girard, Mensonge romantique et vérité romanesque, Hachette Littératures, 2008 [1961], $<$ Pluriel>, p. 21 ; dorénavant $M R$.

11. Jochen Mecke, art. cit.

12. Ibid.

13. Roland Barthes, Fragments d'un discours amoureux, Editions du Seuil, Paris, 1977, <Tel Quel>, p. 271 ; dorénavant $F r$. 


\section{RÉSUMÉS}

Composé de deux romans, Un coup d'un soir et Dans le lit de Marin, le dernier livre de Mathieu Bermann, paru chez POL en 2019, raconte la romance d'un écrivain vivant à Bruxelles et d'un jeune étudiant rennais. L'écrivain narrateur élabore ainsi un roman où il met en scène cette histoire sentimentale et le récit se fait progressivement réflexion sur le désir et sur l'écriture de ce désir. La seconde partie du livre - second roman - constitue cette fiction du désir où se croisent une vérité des émotions, une narration singulière et une réflexion sur la virtualité du désir à partir des échanges sur les réseaux sociaux entre les deux amants. Ce que cherche à faire, simultanément, le personnage écrivain est de produire une œuvre qui ne soit pas vraie, afin de protéger le partenaire officiel du narrateur, Sébastien, de cet adultère secret. De son propre aveu, "c'eût été un mensonge littéraire". Il avoue effectivement, à propos de cette supercherie : "En écrivant, je prenais des risques, qui n'en seraient vraiment qu'au moment de la publication".

Ainsi, l'œuvre de Bermann interroge la vérité de la fiction et du fantasme en jouant sur la virtualité du désir. Le roman écrit par le narrateur dans le roman-cadre devient l'enjeu d'une reconquête du désir, tout en maintenant à distance une réalisation de ce dernier, au seuil du fictif comme mensonge.

\section{INDEX}

Mots-clés : désir, fiction, mensonge, écriture, virtualité

\section{AUTEUR}

\section{RODOLPHE PEREZ}

Université de Tours 